\title{
Pediatric Rapid Response Systems: Identification and Treatment of Deteriorating Children
}

\author{
Amanda B. Levin, $M D^{1,4, *}$ \\ Patrick Brady, MD, MSC ${ }^{2}$ \\ Heather P. Duncan, $M B C h B, M S C, M R C P C H^{3}$ \\ Aisha Barber Davis, $M D^{1}$
}

\author{
Address \\ ${ }^{1}$ Childrens National Health System, 111 Michigan Ave, NW, Washington, DC \\ 20010, USA \\ ${ }^{2}$ Cincinnati Children's Hospital Medical Center, 3333 Burnet Avenue, ML 9016, \\ Cincinnati, $\mathrm{OH}$ 45229, USA \\ ${ }^{3}$ Birmingham Children's Hospital, Steelhouse Lane, Birmingham, B4 6NH, UK \\ ${ }^{*}, 4$ Department of Critical Care Medicine, Children's National Health System, 111 \\ Michigan Avenue, NW, Washington, DC 20010, USA \\ Email: amlevin@childrensnational.org
}

Published online: 10 January 2015

(C) Springer International Publishing AG 2015

This article is part of the Topical Collection on Hospital Medicine

Keywords Medical emergency team - Critical care outreach team - Rapid response team - Pediatric - Early Warning . Calling criteria

\section{Opinion statement}

Rapid response systems (RRSs) aim to identify deteriorating hospitalized patients outside of the intensive care unit, respond quickly, and escalate to a higher level of care if needed. Despite a decade of evaluation, how to best design an RRS is still under study and debate. When considering your RRS, our recommendation is to start with the outcomes: what improvements in patient care are most needed in your environment? These may include reducing cardiac arrest or mortality, reducing critical deterioration, reducing length of stay in intensive care, or avoiding all preventable patient harm. Then, select a strategy for identification of at-risk patients, a response team structure that meets your institution's needs, and a quality improvement and governance structure to ensure you monitor process and outcome variables. The identification limb detects at-risk patients. For this, we recommend an aggregate pediatric early warning score (EWS), clear guidance regarding monitoring type and frequency, flagging of diagnostic risk factors, and a mandatory escalation system that uses the expertise of nurses, patients, and families and works around barriers to enhance response team activation. The structure and function of the 
response limb are also dependent upon the needs of the institution. We recommend a multidisciplinary team with the skills and resources to assess and manage emergencies. Proactive identification through a rover team or scheduled safety huddles may help with earlier identification of at risk patients. The quality improvement and governance structure should be designed around the desired outcomes. Regular monitoring and review of successes, near misses, and failures are vital for the system to improve outcomes.

\section{Introduction}

In recent years, hospitals have adopted rapid response systems (RRSs) to improve the detection of and response to deteriorating patients. Goals of RRSs include reducing cardiopulmonary arrests (CPA) outside of the intensive care unit, reducing mortality, and improving early rescue of deteriorating patients [1]. Failed escalation is involved in $16 \%$ of cases of serious harm. Survival from arrest can be improved from 27 to $80 \%$ if deterioration is recognized earlier [2]. Over the last decade, hospital policy and governance organizations in the USA, UK, Canada, and Australia have endorsed the importance of systems to recognize and respond to clinical deterioration in the hospital [3-6].

An RRS consists of four important elements $[7,8]$ The identification limb detects patient deterioration and makes the call to escalate care. The response limb typically works outside the primary clinical team's chain of command to bring critical care expertise to the patient. The quality improvement limb examines practice and process improvement. Finally, the governance limb provides oversight, communication with hospital leadership, and maintenance of policies and procedures.

\section{Identifying patient deterioration}

Pediatric patients rarely deteriorate suddenly. There may be upwards of $12 \mathrm{~h}$ between the first sign of deterioration and the time interventions are made [9, 10]. Methods of detection include the following: early warning scores (EWS), standard clinical observations (vital signs), electronic monitoring, recognition of risk factors, clinician concern, and parental concern.

The original cardiac arrest or code teams were called when deterioration was detected, often late, and after missed opportunities to rescue the patient early. Delays due to difficulty detecting deterioration and hierarchical escalation processes created the drive to develop new systems to support earlier decision making and treatment $[8,11-13]$. The earliest EWS were described in adults [14] and were followed by pediatric EWS comprised of either single parameter triggers or aggregate scoring systems [12, 15-17]. Trigger criteria are good for screening larger populations and have high sensitivity, but lack adequate specificity, risking alert fatigue [18].

Early warning scores that are aggregate scores sum physiological measurement sub-scores to identify and amplify the trend of physiological deterioration $[9,12,17,19,20,21 \bullet]$. For example, points may be assigned based on vital signs, physical exam findings, diagnoses, or "worrying condition" [18]. Embedding aggregate EWS within the medical record makes it easier for clinicians to see abnormal trends but validity is threatened by miscalculations and 
incomplete or delayed observations $[9,12]$. The prevalence of pediatric EWS in the UK and USA has increased from 20\% in 2005 to $80 \%$ in 2012 [22-24].

Pediatric EWS performance has been tested and compared both retrospectively and prospectively in different data sets. They perform moderately well in predicting cardiopulmonary arrest or call for resuscitation with an area under the receiver operator characteristic scores of 0.73-0.91 [18, 25]. Pediatric EWS have been shown to decrease the time from trigger criteria to clinician response, increase the proportion of deteriorating patients seen by a physician, decrease time to PICU admission, and decrease in PICU length of stay [26]. One study also demonstrated "critical" pediatric EWS values a median of $11 \mathrm{~h}$ and $36 \mathrm{~min}$ prior to a deterioration event, long before care was escalated to a consultant, at a median of $80 \mathrm{~min}$ prior to ICU transfer [9]. However, prospective evaluations of EWS without a response team are rare [12,17]. Qualitatively, clinicians believe that they are beneficial to provide age-appropriate thresholds for sick children, encourage systematic assessments, trigger critical thinking about deterioration management, and support effective communication $[27,28]$. Variability of score types makes meta-analysis and comparison difficult, hence repeated calls for a universal score similar to the National Early Warning Score (NEWs) in the UK [29]. The bedside pediatric early warning system (PEWS) is the most thoroughly validated score to date and is undergoing further evaluation in the multi-site cluster-randomized Evaluating Processes of Care and the Outcomes of Children in Hospital (EPOCH) trial [30].

\section{Standard clinical observations}

Clinicians must see, feel, and judge effectiveness of breathing, skin perfusion, and level of consciousness to identify the deteriorating patient. EWS will only work to protect children from deterioration if frequent, competent assessments are conducted and interpreted correctly and appropriate decisions are made based on that information [31-34]. Practical standards for routine observation and monitoring are scarce as is high-quality research on normative vital signs (VS) values for children [19, 35, 36].

There is a lack of evidence to guide the frequency of assessments and use of behavioral and physiologic indicators of decline in young hospitalized children. Current EWS rely on relatively infrequent (4-12 h) and varied VS measurements, from a choice of up to 36 parameters, on a paper or electronic chart $[18,19,23,29]$. Neurological observations are often excluded or inadequate and monitoring plans are often left to relatively inexperienced clinicians [37]. The effectiveness of the RRS is vulnerable to these flaws and points to opportunities for further research in these areas $[38,39]$.

Normal, abnormal, and normally abnormal vital signs

Physiology changes as children grow as do "normal" VS. Most EWS use expert-derived normal ranges for age $[9,40,41]$. However, recent data suggests that normal distributions for heart and respiratory rate in well and sick hospitalized children are significantly different from published ranges $[41,42 \bullet]$. The thresholds for normal and abnormal heart and respiratory rate should be reconsidered. The rate of change is greatest up 
to the age of 3 years [42・], and age ranges could be better grouped by $0-1,2-3,4-6,7-10$, and $10-18$ years.

Complex patients have their own specific range of normality, e.g., pulse oximetry of $75-85 \%$ is normal in patients with cyanotic heart disease. Using standard thresholds risks over or under reporting physiological deterioration and these unique patients may benefit from customized monitoring plans [43].

\section{Patient monitoring and alerts}

The use of electronic monitoring often complements nursing care of patients. However, there may be flaws or gaps that compromise the quality of this data such as varied electronic monitoring technology, frequency of observations, automated processing of observed data, data mistakes, need for nurses to validate VS, and tailored warnings or alarms to individual patients $[34,44 \bullet, 45 \bullet \bullet, 46,47,48 \bullet]$. Outcomes in patients with continuous electronic monitoring are varied in both pediatrics and adults [49-51]. Technical hurdles to overcome include spurious measurements due to movement, appropriate sensors and battery power, and secure data processing and storage.

Most children who deteriorate to cardiac arrest have co-morbidities [12, 52]. It is possible to identify diagnostic categories, high-risk medications, and laboratory values that on their own can identify which patients are at risk for deterioration or mortality even before the physiological trends are measured. These criteria, summarized in Table 1, describe susceptibility to deterioration based on background health or illness, medication, and age $[12,15,16,44 \bullet, 53-56,57 \bullet$, 58]. The original PEWS score includes additional diagnostic risk criteria that improves performance in comparison to the similar bedside PEWS and the PEW tool (ROC 0.85 vs. 0.75 vs. $0.73)[22,59]$. Similarly, a seven-item non-physiological score identified a group of patients with more than 80 -fold higher probability of deterioration compared to the baseline risk [54]. Current EWS, however, focus on acute, generic physiological abnormalities and do not identify changes from previous observations or worrisome trends. There is a trade-off between simplicity (with better utility/completion) and complexity (with better sensitivity/specificity). Ideally, the background factors should provide a baseline risk and the physiological trends would increase/decrease on that foundation.

Parents of children who have suffered delayed identification of clinical deterioration want to have the ability to call for more expert help when they are seriously concerned about their child [60, 61]. A recent survey identified that this is possible in 60-69 \% of US hospitals and in two states, it is a legal requirement [62-64]. Before implementing parental activation, there were concerns related to resources being overwhelmed by an excess of false alarms, increased parental anxiety, and undermining of therapeutic relationships [65]. However, at one institution with a robust program, there were 42 calls made by concerned parents over a 2-year 
Table 1. Diagnostic and symptomatic indicators of risk for patient deterioration

\begin{tabular}{|c|c|}
\hline Publication & Indicator of increased risk \\
\hline \multirow[t]{10}{*}{ PEWS [12] } & Abnormal airway (not tracheostomy) \\
\hline & Home oxygen \\
\hline & Severe cerebral palsy \\
\hline & Transplant recipient \\
\hline & Gastrostomy tube \\
\hline & Central venous line in situ \\
\hline & Any previous admission to an ICU \\
\hline & Fluid bolus \\
\hline & Increased number of medications per $24 \mathrm{~h}$ \\
\hline & $>3$ medical specialties involved in care \\
\hline \multirow{5}{*}{ Improving situation awareness [44•] } & Family concerns \\
\hline & High-risk therapies \\
\hline & Elevated early warning score \\
\hline & Communication concerns \\
\hline & Watcher/clinician gut feeling \\
\hline \multirow{7}{*}{ Score to predict "critical deterioration" [54] } & Age $<1$ year \\
\hline & Hemoglobin <100 g/L \\
\hline & Congenital/genetic conditions \\
\hline & History of transplant \\
\hline & Enteral tube \\
\hline & Blood cultured in the preceding $72 \mathrm{~h}$ \\
\hline & Epilepsy \\
\hline \multirow[t]{6}{*}{ High risk medications [53] } & $\begin{array}{l}\text { Antibiotics (glycopeptides, third- and fourth- generation cephalosporins, } \\
\text { aminoglycosides) }\end{array}$ \\
\hline & Systemic corticosteroids \\
\hline & Benzodiazepines \\
\hline & Loop diuretics \\
\hline & Narcotic analgesics (full opioid agonists) \\
\hline & Antidotes to hypersensitivity reactions \\
\hline \multirow{12}{*}{$\begin{array}{l}\text { Other diagnostic and symptomatic } \\
\text { indicators of risk }\end{array}$} & Apnea \pm bradycardia $[15,16]$ \\
\hline & Severe respiratory distress $[14,67]$ \\
\hline & Neurologic/seizure disorder, behavior or lethargy $[9,15,16]$ \\
\hline & Postoperative [15] \\
\hline & Persistent vomiting [9] \\
\hline & Cyanosis $[14,58]$ \\
\hline & Congenital/genetic conditions [56] \\
\hline & Signs of shock $[9,16]$ \\
\hline & Racemic epinephrine $[9,16]$ \\
\hline & Hyperkalaemia [16] \\
\hline & Suspected meningococcal disease [16] \\
\hline & Diabetic ketoacidosis [16] \\
\hline
\end{tabular}

period $[61,66]$. Some programs enable parents to activate a rapid response team (RRT) while others are more comprehensive, serving as a format for parents to communicate all concerns to avoid the need for an urgent call $[62,67]$. Educating all families is a challenge; in one study, only $27 \%$ of families understood how to activate the RRT [66]. 


\section{Response limb}

The efferent limb of the RRS consists of a responding team of providers with enough experience to rapidly assess and manage medical emergencies. Singletiered systems have one type of team that responds to all escalation calls, whereas two-tiered systems include a traditional code blue team to respond to CPA and a second team to respond to calls for clinical assessment of deteriorating patients with a goal of reducing CPA outside the ICU [58, 6870]. These teams are typically referred to as rapid response teams (RRTs) or critical care outreach teams (CCOT) if nurse led, medical emergency teams (MET) if physician led, or rover teams if the team has surveillance functions as well as response to calls $[7,11,69,71,72]$. We will use the term RRT. In 2012, $79 \%$ pediatric intensive care units had an RRT, available $24 \mathrm{~h}$ a day, 7 days a week [63]. Physicians were involved in $77 \%$ of their teams [62]. In 2014 UK data, $85 \%$ of units were using PEWS and $18 \%$ had an RRT in place, with rates as high as $52 \%$ for RRT in tertiary hospitals [73].

\section{Evidence for RRT}

The evidence for rapid response teams is strong. A meta-analysis including hundreds of thousands of pediatric patients demonstrates statistically significant $38 \%$ reduction of CPA and $21 \%$ reduction in hospital mortality rates [68]. An updated systematic review by Winters et al., continued to support these findings [ $45 \bullet \bullet]$. There is evidence of reduced respiratory arrest, reduction in the predicted need for ICU level treatments in the first $12 \mathrm{~h}$ of ICU care $[74 \bullet \bullet, 75$, 76] and a reduction in the time between deterioration and treatment from $9 \mathrm{~h}$ $55 \mathrm{~min}$ to $4 \mathrm{~h} 15 \mathrm{~min}$ [10]. The balancing metric is that use of an RRT may lead to an increase in ICU transfers, with one US center noting an increase from 4.5

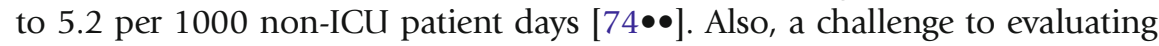
RRTs is the worry that secular trends and other safety interventions may overstate the effect of RRTs. A study at a Canadian hospital without an RRT showed similar declines in mortality as observed in hospitals with RRTs [77]. Still, the level 1 evidence adds weight to the accumulating level 2-5 evidence and is sufficient to support implementation [10, 68, 78•, 79].

Beyond having a RRT, there is evidence in adults for a "dose-response" of RRT with 1 cardiac arrest potentially prevented for every 17 RRT calls [80]. That is, the more RRT calls, the greater reductions in CPA and mortality.

The composition of the RRT team varies according to the availability of providers and institutional needs $[8,61,62]$. Traditionally, pediatric RRTs include a combination of medical (critical care attending or fellow hospitalist, resident), critical care nurse, and respiratory therapist. Some teams for adult patients also include laboratory technicians, radiology technician, pharmacist, electrocardiography technician, phlebotomists, and physician's assistants [81, 82]. Traditionally, pediatric critical care physicians have led RRTs with hospitalists, nurse practitioners, and nurses less commonly, particularly for systems with a second- 
tier team. One study reports that $29 \%$ of hospitals with a PICU had RRTs led by nurses [61].

Another consideration is whether providers are staffed solely for the purposes of the RRS or have additional clinical responsibilities. In a survey of US children's hospitals, the majority of the teams included nurses with other clinical responsibilities outside of the RRS [64]. However, several hospitals noted that there were significant concerns regarding adequate staffing of the ICU when nurses were out of the unit responding to calls from the wards. Optimal team composition of the pediatric RRT is not known $[45 \bullet \bullet]$.

RRTs should have the skills and resources to assess and stabilize the patient at the bedside in collaboration with the primary team. They should be prepared to perform common interventions required of RRTs including airway clearance, oxygen administration, respiratory medications, positivepressure ventilation by bag-valve mask, interpret chest X-rays, gain intravenous access, administer rapid fluid resuscitation, measure blood glucose, and provide other necessary medications [83, 84]. Non-technical skills of RRT members including leadership and communication skills are also important, with evidence in both adult and pediatric settings [28, 78•, $85,86]$.

Qualitative benefits

The benefits of RRTs go beyond patient outcomes. RRTs empower providers to call for help and help to break down hierarchal culture [75, 87]. Nursing education may improve activation of the RRT and lead to decreases in code rates $[78 \bullet, 86]$. A qualitative review of the adult nursing literature on effective use of an RRT found nurses with more training or expertise felt more confident to activate but education and exposure to RRTs improves confidence in junior nurses [88]. The pediatric literature supports that RRTs promote self-efficacy, the benefits of teaming up with others, provide objective criteria to activating an RRT, provides support to inexperienced clinician, and helps overcome hierarchical norms [28].

There are on-going barriers to acceptance and effective implementation of a RRS. Proper escalation of care is one area of concern. A multi-centered study of adult patients in Australian hospitals reported $42 \%$ of events with appropriate triggers did not result in an RRT call, even in settings with wide-spread institutional support [89]. Other adult literature report that some may still view RRTs as not effective or life-saving, lead to poor communication in silos of care, and create of additional workload to ICU personnel [13, 88, 90]. A pediatric study of attitudes and barriers reported that $32 \%$ of nurse and $47 \%$ of doctors would not activate an RRT for a patient fulfilling criteria if the patient appeared well. Yet, $30 \%$ of doctors and $15 \%$ of nurses had failed to activate an RRT when needed [91]. The reasons for not escalating concerns about deterioration may be lack of face validity, lack of confidence or expertise, or cultural barriers. In a pediatric study, cultural and socio-political reasons identified include the following: 
1. Self-efficacy in recognizing deteriorating conditions and activating the RRT

2. Intraprofessional and interprofessional hierarchies

3. Expectations of adverse interpersonal or clinical outcomes from RRT activations and intensive care unit transfers (e.g., reluctance among subspecialty attending physicians to transfer patients to the intensive care unit for fear of inappropriate management) [28]

Additional barriers include low morale and lack of teamwork, fear of criticism, negative attitudes of attendees, lack of education, and workload [90, 91].

National policy statements specifically recommend identifying and managing the local barriers to implementation of systems including reliable escalation at the patient level [3, 4, 92-94]. Optimizing commitment of staff at senior level, governance arrangements, technological support, sharing of information, especially successes, and effective education and training of staff address these barriers.

\section{Quality improvement and governance}

The RRS and RRTs are complex interventions that require both improvement and governance oversight to ensure they effectively meet their safety and quality aims. This is best accomplished by an inter-professional team that includes clinical leaders: ideally, physicians both based on the ICU and in areas with a high number of RRT activations, nursing in the same areas, and respiratory therapy. In the early stages it is valuable to have a data analyst with expertise in run and control chart creation and a member with improvement science expertise. While the expertise needed to administer and improve an RRS has some overlap with the expertise needed to oversee a code team, it may be of value to have a separate team or working group charged with RRS oversight.

In both the implementation and monitoring phase, it is important to track three types of metrics to evaluate the success of the team and identify opportunities for improvement: outcome measures, process measures, and balancing measures. Outcome measures are best described in the literature and the most commonly tracked are codes outside of the ICU $[2,58]$. Two important challenges are of note with codes outside the ICU as a primary outcome measure. First and most importantly, code events are a small subset of code team activations, and it may be difficult to demonstrate improvement in a single center. The code rate in a recent systematic review was 5.9 per 10,000 patients, a sufficiently low number to make it difficult to detect a downward change at any one center without many years of data [68]. Secondly, as certain types of code events (e.g., a first-time seizure that leads to apnea) are likely not preventable by an RRS, a goal of zero codes may not be achievable [67]. The use of a preventable code metric has the advantage of setting a zero goal which may be more motivating for the 
organization, but determining post hoc preventability is difficult, particularly in a way that might generalize. Codes outside of the ICU do represent potential sentinel events and may offer information on failure modes or risks in the activation or response of the RRT. In-hospital mortality is also a frequently used and important metric but has two challenges. Mortality is rare in children's hospitals, and it is likely driven by factors that are amenable to RRS and those that are not (e.g., quality of ICU care).

In the last several years, three proximate metrics have been developed to assess the efficacy of pediatric RRS: critical deterioration [57•], unrecognized situation awareness failure events (UNSAFE transfers) [44•], and transfer requiring intensive practice (TRIP) events [95]. Critical deterioration is defined as any patient who transfers from the floor to the ICU and receives vasopressors and is intubated or placed on non-invasive pressure support within $12 \mathrm{~h}$. This is the best validated of the proximate measures. Critical deterioration occurs more than eight times more commonly than codes outside the ICU and is associated with a greater than 13 -fold increase in mortality. The initiation of an RRS at the Children's Hospital of Philadelphia was associated with a significant reduction in the occurence of critical deterioration events [76]. As critical deterioration captures some children in whom CPAP is begun $10 \mathrm{~h}$ into an ICU stay, it is likely a more sensitive and less specific marker of RRS-preventable deterioration. Alternatively, UNSAFE transfers, defined as a patient transferred from the floor to the ICU who is intubated, placed on vasopressors or given three or more fluid boluses within $1 \mathrm{~h}$ of ICU transfer, is a more specific and less sensitive measure [44•]. A complex intervention targeting improved situation awareness at Cincinnati Children's reduced the rate of UNSAFE transfers by $75 \%$ [44•]. A similar proximate measure is the TRIP developed at Seattle Children's [95]. A TRIP is a transfer from the hospital floor to the ICU who requires intubation, non-invasive pressure support, or vasopressors within $2 \mathrm{~h}$ of ICU transfer. Early evaluation has shown reasonable sensitivity and specificity for survival at 28 days.

Several process measures are useful in evaluating that the RRS is functioning as intended. The number of calls RRT calls placed per day or per month is a valuable metric for several reasons. First, the number of calls placed is a useful proxy measure for how well the identification limb is functioning. This includes both the function of specific triggering mechanisms such as EWS calculations and triggering as well as culture on individual units. Second, quantitative data that a unit has not called an RRT in months may be valuable in identifying an area where clinicians do not support calling the RRT. Conversely, high number of calls may identify training opportunities for unit staff. This may be considered in the context of a dose-response as hospitals with mature RRTs that report improved patient outcome following RRT introduction have a RRT dose between 25.8 and 56.4 calls per 1000 admissions. The concern relates to a low dose reflecting a dysfunctional afferent limb or safety culture. Other process measures include the proportion of RRT calls transferred to the ICU, RRT calls stratified by time of day and day of week, response time of the RRT (15-30 min is most common), and time between elevated PEWS and RRT evaluation or ICU transfer (socalled score to door time) [96]. Similar to the number of calls, the right 
number is uncertain and likely dependent on organizational factors such as number of ICU beds, but our hospitals consistently have $50-60 \%$ of RRT calls transferred within $4 \mathrm{~h}$ of call.

The most commonly discussed balancing measures for RRTs based in the ICU is the cost of taking expert critical care clinicians away from the ICU. This cost is difficult to measure and has not been demonstrated in any studies, but it is an important issue for RRS leaders to consider when deciding the composition of the team. Since RRS function across silos, it may be unclear which departments should finance which aspect of the system. In terms of preventing adverse events, the cost-benefit of an RRT at one large US children's hospital has recently demonstrated that a RRT composed of clinicians with concurrent responsibilities would recoup its value with preventing only 3.5 critical deteriorations

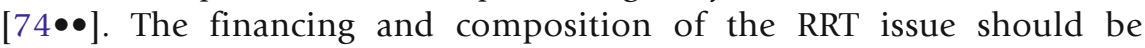
addressed from the onset of the team and may require a financial

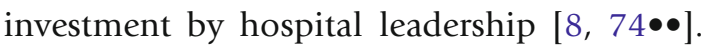

\section{Administrative and governance}

\section{Summary}

The administrative and governance limb of an RRS has the job of coordinating resources, finances, and relationships with hospital leadership, risk management, information technology, and patient safety. RRS encounter many barriers and benefit from the support of nursing and medical leadership for sustainability [8]. Interaction with hospital leadership is important for garnering buy-in, financing, and support for educational efforts, and quality improvement initiatives. A reporting structure to hospital leadership can also enhance safety through facilitating reporting of identified latent safety threats or other patient care, safety, or staffing issues revealed during RRS activations or process evaluation. A predetermined reporting structure is important to facilitating this.

Rapid response systems are comprehensive, hospital-wide systems of care that can effectively reduce harm and mortality to children. RRSs have an accepted, internationally recognized framework of identification, response, quality improvement, and governance arms and strong support from national safety bodies. Many before and after studies and two systematic reviews have demonstrated the utility of EWS and RRT to reduce cardiopulmonary arrest and mortality, new data endorses their cost-effectiveness, and emerging clinical deterioration metrics allow for further study and improvement. More evidence is needed for optimal patient monitoring, adoption of appropriate vital signs for sick inpatients, the ideal make up of an RRT, and the financial case for an RRS. Hospitals must address threats to the success of an RRS including cultural barriers, hierarchy, punitive consequences for calling the team, lack of appropriate education and training, staffing concerns, leadership, and financial support. The case for implementing an RRS in pediatric hospitals is made; how best to do this is evolving. 


\section{Compliance with Ethics Guidelines}

\section{Conflict of Interest}

Amanda B. Levin, Patrick Brady, Heather Duncan, and Aisha Barber Davis declare that they have no conflict of interest.

\section{Human and Animal Rights and Informed Consent}

This article does not contain any studies with human or animal subjects performed by any of the authors.

\section{References and Recommended Reading}

Papers of particular interest, published recently, have been highlighted as:

- Of importance

$\bullet \quad$ Of major importance

1. Institute for Healthcare Improvement: 5 Million Lives Campaign. Getting Started Kit: Rapid Response Teams. 2008. (www.IHI.org/).

2. Hayes LW, Dobyns EL, DiGiovine B, Brown AM, Jacobson S, Randall KH, et al. A multicenter collaborative approach to reducing pediatric codes outside the ICU. Pediatr. 2012;129(3):e785-91.

3. The Australian Commission on Safety and Quality in healthcare: National Consensus Statement, essential elements for recognizing and responding to clinical deterioration. 2008. (www.safetyandquality.gov.au/ wp-content/uploads/2011/09/NSQHSStandards).

4. National Institute for Health and Clinical Excellence. Acutely Ill Patients in Hospital: Recognition of and response to acute illness in adults in hospital. 2007. (http://www.nice.org.uk/nicemedia/pdf/ CG50FullGuidance.pdf).

5. The Joint Commission announces the 2008 National Patient Safety Goals and Requirements. Joint Comm Perspect Joint Comm Accred Healthc Organ 2007, 27(7):1, 9-22.

6. Ontario Ministry of Health and Long-term Care: Critical Care Strategy (www.health.gov.on.ca/en/pro/ programs/criticalcare/strategy.aspx).

7. Devita MA, Bellomo R, Hillman K, Kellum J, Rotondi A, Teres D, et al. Findings of the first consensus conference on medical emergency teams. Crit Care Med. 2006;34(9):2463-78.

8. Jones DA, DeVita MA, Bellomo R. Rapid-response teams. N Engl J Med. 2011;365(2):139-46.

9. Akre M, Finkelstein M, Erickson M, Liu M, Vanderbilt L Billman G. Sensitivity of the pediatric early warning score to identify patient deterioration. Pediatr. 2010;125(4):e763-9.

10. Hanson CC, Randolph GD, Erickson JA, Mayer $\mathrm{CM}$, Bruckel JT, Harris BD, et al. A reduction in cardiac arrests and duration of clinical instability after implementation of a paediatric rapid response system. Postgrad Med J. 2010;86(1015):314-8.

11. Tibballs J, van der Jagt EW. Medical emergency and rapid response teams. Pediatr Clin North Am. 2008;55(4):989-1010. xi.

12. Duncan H, Hutchison J, Parshuram CS. The pediatric early warning system score: a severity of illness score to predict urgent medical need in hospitalized children. J Crit Care. 2006;21(3):271-8.

13. Devita M, Hillman K. Potential sociological and political barriers to medical emergency team implementation. In: Medical emergency teams. New York: Springer; 2006.

14. McNeill G, Bryden D. Do either early warning systems or emergency response teams improve hospital patient survival? A systematic review. Resuscitation. 2013;84:1652-67.

15. Tibballs J, Kinney S. Reduction of hospital mortality and of preventable cardiac arrest and death on introduction of a pediatric medical emergency team. Pediat Crit Care Med: A J Soc Crit Care Med World Fed Pediat Intensiv Crit Care Soc. 2009;10(3):306-12.

16. Haines C, Perrott M, Weir P. Promoting care for acutely ill children-development and evaluation of a paediatric early warning tool. Intensiv Crit Care Nurs: Off J $\mathrm{Br}$ Assoc Crit Care Nurses. 2006;22(2):73-81.

17. Parshuram CS, Bayliss A, Reimer J, Middaugh K, Blanchard N. Implementing the bedside paediatric early warning system in a community hospital: a prospective observational study. Paediatr Child Health. 2011;16(3):e18-22.

18. Robson MA, Cooper CL, Medicus LA, Quintero MJ, Zuniga SA. Comparison of three acute care pediatric early warning scoring tools. J Pediatr Nurs. 2013;28(6):e33-41.

19. Standards for assessing, measuring, and monitoring vital signs in infants, children, and young people. In. RCN guidance for children's nurses and nurses working with children and young people: Royal College of Nursing; 2011.

20. Parshuram CS, Duncan HP, Joffe AR, Farrell CA Lacroix JR, Middaugh KL, et al. Multicentre validation 
of the bedside paediatric early warning system score: a severity of illness score to detect evolving critical illness in hospitalised children. Crit Care. 2011;15(4):R184.

21. Pearson GD, Heather D. Early warning systems for identifying sick children. Paediatr Child Health. 2011;21(5):230-3.

Class III: This study is a retrospective case-control study evaluating three commonly used pediatric EWS.

22. Are We There Yet? A review of organisational and clinical aspects of children's surgery. In. National Confidential Enquiry into Patient Outcome and Death; 2011. (www.ncepod.org.uk/2011report1/downloads/ SIC_fullreport.pdf).

23. Duncan HP. Survey of early identification systems to identify inpatient children at risk of physiological deterioration. Arch Dis Child. 2007;92(9):828.

24. VandenBerg SD, Hutchison JS, Parshuram CS. A crosssectional survey of levels of care and response mechanisms for evolving critical illness in hospitalized children. Pediatr. 2007;119(4):e940-6.

25. Chapman SM, Grocott MP, Franck LS. Systematic review of paediatric alert criteria for identifying hospitalised children at risk of critical deterioration. Intensiv Care Med. 2010;36(4):600-11.

26. Theilen UL, P; Agrawal, D; Weitz, J; Simpson, D. Impact of early warning on paediatric intensive care. Arch Dis Child 2010; 95(Supp 1).

27. Bonafide $\mathrm{CP}$, Roberts KE, Weirich CM, Paciotti B, Tibbetts KM, Keren R, et al. Beyond statistical prediction: qualitative evaluation of the mechanisms by which pediatric early warning scores impact patient safety. J Hosp Med: Off Publ Soc Hosp Med. 2013;8(5):248-53.

28. Roberts KE, Bonafide CP, Paine CW, Paciotti B, Tibbetts $\mathrm{KM}$, Keren R, et al. Barriers to calling for urgent assistance despite a comprehensive pediatric rapid response system. Am J Crit Care. 2014;23(3):223-9.

29. National Early Warning Score (NEWS). Standardising the assessment of acute illness severity in the NHS. In. Report of a working party. Royal College of Physicians 2012.

30. The Hospital for Sick Children. Evaluating Processes of Care \& the Outcomes of Children in Hospital (EPOCH) http://clinicaltrials.gov/show/ NCT01260831 (http://clinicaltrials.gov/show/ NCT01260831).

31. Preston R, Flynn D. Observations in acute care: evidence-based approach to patient safety. Br J Nurs. 2010;19(7):442-7.

32. Thomson RL, Beaumont D, Healey K, Scrobie FS. Safer care for the acutely ill patient: learning from serious incidents. In: The fifth report from the Patient Safety Observatory. National Patient Safety Agency 2007.

33. Harley S, Timmons S. Clinical assessment skills and the use of monitoring equipment. Paediatr Nurs. 2010;22(8):14-8.

34. Liaw SY, Scherpbier A, Klainin-Yobas P, Rethans JJ. A review of educational strategies to improve nurses' roles in recognizing and responding to deteriorating patients. Int Nurs Rev. 2011;58(3):296-303.
35. McCabe A, Duncan H. National survey of observation and monitoring practices of children in hospital. Paediatr Nurs. 2008;20(6):24-7.

36. Van Kuiken D, Huth MM. What is 'normal?' evaluating vital signs. Pediatr Nurs. 2013;39(5):216-24.

37. Watson A, Skipper C, Steury R, Walsh H, Levin A. Inpatient nursing care and early warning scores: a workflow mismatch. J Nurs Care Qual.

2014;29(3):215-22.

38. Hands C, Reid E, Meredith P, Smith GB, Prytherch DR, Schmidt PE, et al. Patterns in the recording of vital signs and early warning scores: compliance with a clinical escalation protocol. BMJ Qual Saf. 2013;22(9):719-26.

39. Andrews T, Waterman H. Packaging: a grounded theory of how to report physiological deterioration effectively. J Adv Nurs. 2005;52(5):473-81.

40. Edwards ED, Mason BW, Oliver A, Powell CV. Cohort study to test the predictability of the Melbourne criteria for activation of the medical emergency team. Arch Dis Child. 2011;96(2):174-9.

41. Fleming S, Thompson M, Stevens R, Heneghan C, Pluddemann A, Maconochie I, et al. Normal ranges of heart rate and respiratory rate in children from birth to 18 years of age: a systematic review of observational studies. Lancet. 2011;377(9770):1011-8.

42.• Bonafide CP, Brady PW, Keren R, Conway PH, Marsolo $\mathrm{K}$, Daymont C. Development of heart and respiratory rate percentile curves for hospitalized children. Pediatr. 2013;131(4):e1150-7.

Class III Evidence: This study is a inpatient cohort study determining new inpatient vital sign norms and calling into question previous standards.

43. McLellan MC, Connor JA. The Cardiac Children's Hospital Early Warning Score (C-CHEWS). J Pediatr Nurs. 2013;28(2):171-8.

44. $\quad$ Brady PW, Muething S, Kotagal U, Ashby M, Gallagher $\mathrm{R}$, Hall D, et al. Improving situation awareness to reduce unrecognized clinical deterioration and serious safety events. Pediatr. 2013;131(1):e298-308.

Class III Evidence - This is a quality improvement study introducing a new metric of deterioration, UNSAFE Transfer, and demonstrates improvement in this metric through iterative quality improvement initiatives.

45.• Winters BD, Pham JC, Hunt EA, Guallar E, Berenholtz S, Pronovost PJ. Rapid response systems: a systematic review. Crit Care Med. 2013;35(5):1238-43.

Class I Evidence - This study is an update to a 2010 Metaanalysis of RRS, demonstrating decreased non-ICU arrest and total hospital mortality.

46. Nangalia V, Prytherch DR, Smith GB. Health technology assessment review: remote monitoring of vital signs-current status and future challenges. Crit Care. 2010;14(5):233.

47. Churpek MM, Yuen TC, Park SY, Gibbons R, Edelson DP. Using electronic health record data to develop and validate a prediction model for adverse outcomes in the wards*. Crit Care Med. 2014;42(4):841-8.

48. Zhai H, Brady P, Li Q, Lingren T, Ni Y, Wheeler DS, et al. Developing and evaluating a maching learning 
based algorithm to predict the need of peidatric intensive care unit transfer for newly hospitalized children. Resuscitation. 2014;85(8):1065-71.

Class III: This is a case-control study of a novel eletronic medical record algorithm that performed better than two pediatric EWS reported in the literature.

49. Panesar R, Polikoff LA, Harris D, Mills B, Messina C, Parker MM. Characteristics and outcomes of pediatric rapid response teams before and after mandatory triggering by an elevated Pediatric Early Warning System (PEWS) score. Hosp Pediatr. 2014;4(3):135-40.

50. Jones CM, Bleyer AJ, Petree B. Evolution of a rapid response system from voluntary to mandatory activation. Joint Comm J Qual Patient Saf / Joint Comm Res 2010, 36(6):266-270, 241.

51. Huh JW, Lim CM, Koh Y, Lee J, Jung YK, Seo HS, et al. Activation of a medical emergency team using an electronic medical recording-based screening system*. Crit Care Med. 2014;42(4):801-8.

52. Girotra S, Spertus JA, Li Y, Berg RA, Nadkarni VM, Chan PS. Survival trends in pediatric in-hospital cardiac arrests: an analysis from get with the guidelinesresuscitation. Circ Cardiovasc Qual Outcomes. 2013;6(1):42-9.

53. Huang EJ, Bonafide CP, Keren R, Nadkarni VM, Holmes JH. Medications associated with clinical deterioration in hospitalized children. J Hosp Med: Off Publ Soc Hosp Med. 2013;8(5):254-60.

54. Bonafide CP, Holmes JH, Nadkarni VM, Lin R, Landis JR, Keren R. Development of a score to predict clinical deterioration in hospitalized children. J Hosp Med: Off Publ Soc Hosp Med. 2012;7(4):345-9.

55. Mohammed MA, Rudge G, Wood G, Smith G, Nangalia V, Prytherch D, et al. Which is more useful in predicting hospital mortality-dichotomised blood test results or actual test values? A retrospective study in two hospitals. PLoS One. 2012;7(10):e46860.

56. Bion JF. Susceptibility to critical illness: reserve, response and therapy. Intensiv Care Med. 2000;26 Suppl 1:S57-63.

57. $\quad$ Bonafide CP, Roberts KE, Priestley MA, Tibbetts KM, Huang E, Nadkarni VM, et al. Development of a pragmatic measure for evaluating and optimizing rapid response systems. Pediatr. 2012;129(4):e874-81.

Class III Evidence - This study defines a new metric of deterioration that occurs at a 13-fold higher rate than mortality, is a proximate outcome associated with in-hospital mortality, and can be useful as a metric for RRS quality improvement.

58. Brilli RJ, Gibson R, Luria JW, Wheeler TA, Shaw J, Linam $M$, et al. Implementation of a medical emergency team in a large pediatric teaching hospital prevents respiratory and cardiopulmonary arrests outside the intensive care unit. Pediatr Crit Care Med: J Soc Crit Care Med World Fed Pediatr Intensiv Crit Care Soc. 2007;8(3):236-46.

59. Parshuram CS, Hutchison J, Middaugh K. Development and initial validation of the bedside paediatric early warning system score. Crit Care.

2009;13(4):R135.
60. REACH Parental Activation (www.cec.health.nsw.gov. $\mathrm{au} /$ documents/programs/partnering-with-patients/ pbcc/attachment/4-2-reach-toolkit.pdf).

61. Dean BS, Decker MJ, Hupp D, Urbach AH, Lewis E, Benes-Stickle J. Condition HELP: a pediatric rapid response team triggered by patients and parents. J Healthc Qual. 2008;30(3):28-31.

62. Chen JG, Kemper AR, Odetola F, Cheifetz IM, Turner DA. Prevalence, characteristics, and opinions of pediatric rapid response teams in the United States. Hosp Pediatr. 2012;2(3):133-40.

63. Sen AI, Morgan RW, Morris MC. Variability in the implementation of rapid response teams at academic American pediatric hospitals. J Pediatr.

2013;163(6):1772-4.

64. Smith J. Crossing the Quality Chasm: Patient and Family Activated Rapid Response Methods. In: University of Houston Law Center 2009.

65. Paciotti B, Roberts KE, Tibbetts KM, Paine CW, Keren R, Barg FK, et al. Physician attitudes toward familyactivated medical emergency teams for hospitalized children. Joint Comm J Qual Patient Saf / Joint Comm Resour. 2014;40(4):187-92.

66. Ray EM, Smith R, Massie S, Erickson J, Hanson C, Harris B, et al. Family alert: implementing direct family activation of a pediatric rapid response team. Joint Comm J Qual Patient Saf / Joint Comm Resour. 2009;35(11):575-80.

67. Honey L, Montgomery, H: Shine 2012 Final Report: Listening to you. 2012.

68. Chan PS, Jain R, Nallmothu BK, Berg RA, Sasson C. Rapid response teams: a systematic review and metaanalysis. Arch Intern Med. 2010;170(1):18-26.

69. DeVita MA, Bellomo R, Hillman K. Introduction to the rapid response systems series. Joint Comm J Qual Patient Saf / Joint Comm Resour. 2006;32(7):35960.

70. Nowak JE, Brilli RJ. Pediatric rapid response teams: is it time? Jama. 2007;298(19):2311-2.

71. Sakai T, Devita MA. Rapid response system. J Anesth. 2009;23(3):403-8.

72. Hueckel RM, Turi JL, Cheifetz IM, Mericle J, Meliones JN, Mistry KP. Beyond rapid response teams: instituting a "Rover Team" improves the management of at-risk patients, facilitates proactive interventions, and improves outcomes advances in patient safety: new directions and alternative approaches (Vol. 3: Performance and Tools). Rockville MD 2008.

73. Roland D, Oliver A, Edwards ED, Mason BW, Powell $\mathrm{CV}$. Use of paediatric early warning systems in Great Britain: has there been a change of practice in the last 7 years? Arch Dis Child. 2014;99(1):26-9.

74.• Bonafide CP, Localio AR, Song L, Roberts KE, Nadkarni VM, Priestley M, et al. Cost-benefit analysis of a medical emergency team in a children's hospital. Pediatr. 2014;134(2):235-41.

Class III Evidence: This study is a cohort study matching clinical and administrative data that demonstrates a cost-benefit of RRTs in a children's hospital. 
75. Hunt EA, Zimmer KP, Rinke ML, Shilkofski NA, Matlin $\mathrm{C}$, Garger $\mathrm{C}$, et al. Transition from a traditional code team to a medical emergency team and categorization of cardiopulmonary arrests in a children's center. Arch Pediatr Adolesc Med. 2008;162(2):117-22.

76. Bonafide CP, Localio AR, Roberts KE, Nadkarni VM, Weirich CM, Keren R. Impact of rapid response system implementation on critical deterioration events in children. JAMA Pediatr. 2014;168(1):25-33.

77. Joffe AR, Anton NR, Burkholder SC. Reduction in hospital mortality over time in a hospital without a pediatric medical emergency team: limitations of beforeand-after study designs. Arch Pediatr Adolesc Med. 2011;165(5):419-23.

78. Theilen U, Leonard P, Jones P, Ardill R, Weitz J, Agrawal $\mathrm{D}$, et al. Regular in situ simulation training of paediatric medical emergency team improves hospital response to deteriorating patients. Resuscitation. 2013;84(2):218-22.

Class IV Evidence - This study demonstrated that regular team training on hospital response to deteriorating patients reduced time to recognition of deterioration, decreasing the proportion of patients with $>12$ h delay in recognition from $23 \%$ to $2 \%$ and reduced median time of recognition of deterioration from $11 \mathrm{~h}$ to $1.5 \mathrm{~h}$.

79. Tee A, Calzavacca P, Licari E, Goldsmith D, Bellomo R. Bench-to-bedside review: the MET syndrome-the challenges of researching and adopting medical emergency teams. Crit Care. 2008;12(1):205.

80. Jones D, Bellomo R, DeVita MA. Effectiveness of the medical emergency team: the importance of dose. Crit Care. 2009;13(5):313.

81. Butner SC. Rapid response team effectiveness. Dimens Crit Care Nurs. 2011;30(4):201-5.

82. Buttfield MA, Amos JD, Hillman KM. The medical emergency team as a safety net. Joint Comm J Qual Patient Saf / Joint Comm Resour. 2006;32(11):641-5.

83. Van Voorhis KT, Willis TS. Implementing a pediatric rapid response system to improve quality and patient safety. Pediatr Clin North Am. 2009;56(4):919-33.

84. Anwar ul H, Saleem AF, Zaidi S, Haider SR. Experience of pediatric rapid response team in a tertiary care hospital in Pakistan. Indian J Pediatr. 2010;77(3):273-6.

85. Chalwin RP, Flabouris A. Utility and assessment of non-technical skills for rapid response systems and medical emergency teams. Intern Med J.

2013;43(9):962-9.
86. Famolare N, Romano JC. The implementation of PEARS training: supporting nurses in non-critical care settings to improve patient outcomes. J Pediatr Nurs. 2013;28(3):267-74.

87. Zenker P, Schlesinger A, Hauck M, Spencer S, Hellmich $\mathrm{T}$, Finkelstein $\mathrm{M}$, et al. Implementation and impact of a rapid response team in a children's hospital. Joint Comm J Qual Patient Saf / Joint Comm Resour. 2007;33(7):418-25.

88. Jones L, King L, Wilson C. A literature review: factors that impact on nurses' effective use of the Medical Emergency Team (MET). J Clin Nurs.

2009;18(24):3379-90.

89. Shearer B, Marshall S, Buist MD, Finnigan M, Kitto S, Hore $\mathrm{T}$, et al. What stops hospital clinical staff from following protocols? An analysis of the incidence and factors behind the failure of bedside clinical staff to activate the rapid response system in a multi-campus Australian metropolitan healthcare service. BMJ Qual Saf. 2012;21(7):569-75.

90. DeVita MA, Smith GB, Adam SK, Adams-Pizarro I, Buist M, Bellomo R, et al. "Identifying the hospitalised patient in crisis"-a consensus conference on the afferent limb of rapid response systems. Resuscitation. 2010;81(4):375-82.

91. Azzopardi P, Kinney S, Moulden A, Tibballs J. Attitudes and barriers to a medical emergency team system at a tertiary paediatric hospital. Resuscitation.

2011;82(2):167-74.

92. Pearson G. Why Children Die: the report of a pilot confidential enquiry into child death by CEMAH (Confidential Enquiry into Maternal and Child Health) In. 2008.

93. Kotsakis A, Lobos AT, Parshuram C, Gilleland J, Gaiteiro R, Mohseni-Bod H, et al. Implementation of a multicenter rapid response system in pediatric academic hospitals is effective. Pediatr. 2011;128(1):72-8.

94. National Safety and Quality Health Service Standards (www.safetyandquality.gov.au/publications/nationalsafety-and-quality-health-service-standards).

95. Roberts JM, Leininger WE, Clifton RJ, H. Development of Definition of Failure To Recognize and Respond in Pediatric Inpatients. In: Pediatric Academic Society; Vancouver; 2014.

96. Oglesby KJ, Durham L, Welch J, Subbe CP. 'Score to Door Time', a benchmarking tool for rapid response systems: a pilot multi-centre service evaluation. Crit Care. 2011;15(4):R180. 